\title{
INTEGRATION OF SOFTWARE SOLUTIONS VIA AN INTERMEDIARY WEB SERVICE
}

\author{
P. Milev* \\ University of National and World Economy, Sofia, Bulgaria
}

\begin{abstract}
The article examines issues, related to the integration of software solutions. In this sense, the paper presents features of information systems with multilayered architecture. The integration of the individual physical layers of the system is the most important issue for these software solutions. The main purpose of the article is to explore the possibilities for realization of such integration and to present a conceptual model for communication between the different layers of an information system through an intermediary web service. To achieve its goal, the article uses various scientific methods, including study, analysis, research, modeling and experimentation. The results of this paper support the main hypothesis of the study, namely defining the advantages of integrating information systems with the help of an intermediary software service. The conclusion highlights the possibilities for improvement of communication between different physical layers of software solutions with distributed business logic.
\end{abstract}

Key words: Information system, business logic, distributed application, conceptual model, software architecture.

\section{INTRODUCTION}

Nowadays, the number of software solutions worldwide is significant. Most of them are web-based information systems. There are also many desktop applications that implement a specific business logic. Mobile applications are also increasing. The number of software solutions has grown steadily. Very often, some of these software solutions need to exchange data with each other. The integration of information systems has always been an actual topic. Nowadays it is more than essential. The need of integration of software solutions can be seen from several different aspects:

- From the point of view of the possibility that different software solutions work with common data;

- From the point of view of the possibility that a software solution receives data from another software solution;

- From the point of view of the possibility to divide the business logic of a software

Correspondence to: Plamen Milev, University of National and World Economy, Sofia 1700, tel. +35928195 312,e-mail: pmilev@unwe.bg solution into several parts, which must be connected to one another (to be able to communicate through a protocol).

The need for integration that is generated by the work of various software solutions with common data is frequent in various information systems scenarios. This is the case for universities with a large amount of data that are used by different university systems. Many authors explore these particularities [1]. In the studies of other authors, the emphasis is placed on a large part of public systems, which also work with common data registers $[2,3]$. In any case, the data should first be properly analyzed and then integrated into the decision-making process for organizational purposes [4]. It is logical to suppose that more data would lead to better results in the work of software analysis solutions and therefore better management decisions in organizations. In this sense, the necessity of choosing the right approach for integration of software solutions is of paramount importance. The need for integration that is generated by the exchange of 
data between different software solutions is common in the scenarios of the so-called web services. Web service is a way of transferring data from one software solution to another. Nowadays, many authors are exploring the development of web services in the form of cloud infrastructure as a service [5]. The need for integration that is generated by the separation of the business logic of an information system into separate logical and physical layers is also common nowadays. The distributed business logic of information systems has long been of interest to the research of various authors [6]. This study will first examine traditional approaches for integration of software solutions and then explain the need of application of an additional layer for systems integration.

\section{TRADITIONAL APPROACH FOR INTEGRATION OF SOFTWARE SOLUTIONS}

Approaches for integration of software solutions are in the focus of many authors who are exploring this need. According to some of them, the main problems in the context of the realization of such integration are the many different types of systems, the lack of a uniform format of data, the interconnection with other systems, management issues, etc. [7]. The right management approaches also highlight the possibilities for systems integration [8]. The integration of software solutions is most often implemented with the help of the so-called integration layer. It is a programming interface that serves as an intermediate component. Figure 1 illustrates this approach. On one side of the integration layer is the software solution that provides this integration layer. On the other side of the integration layer are the software solutions that want to make queries to the first software solution. These queries are served by the integration layer. It plays the role of a firewall between the first software solution and the other (additional) software solutions. In this way the data of the first software solution is protected by this firewall and the access to it is limited according to the rules on which the integration layer is implemented.

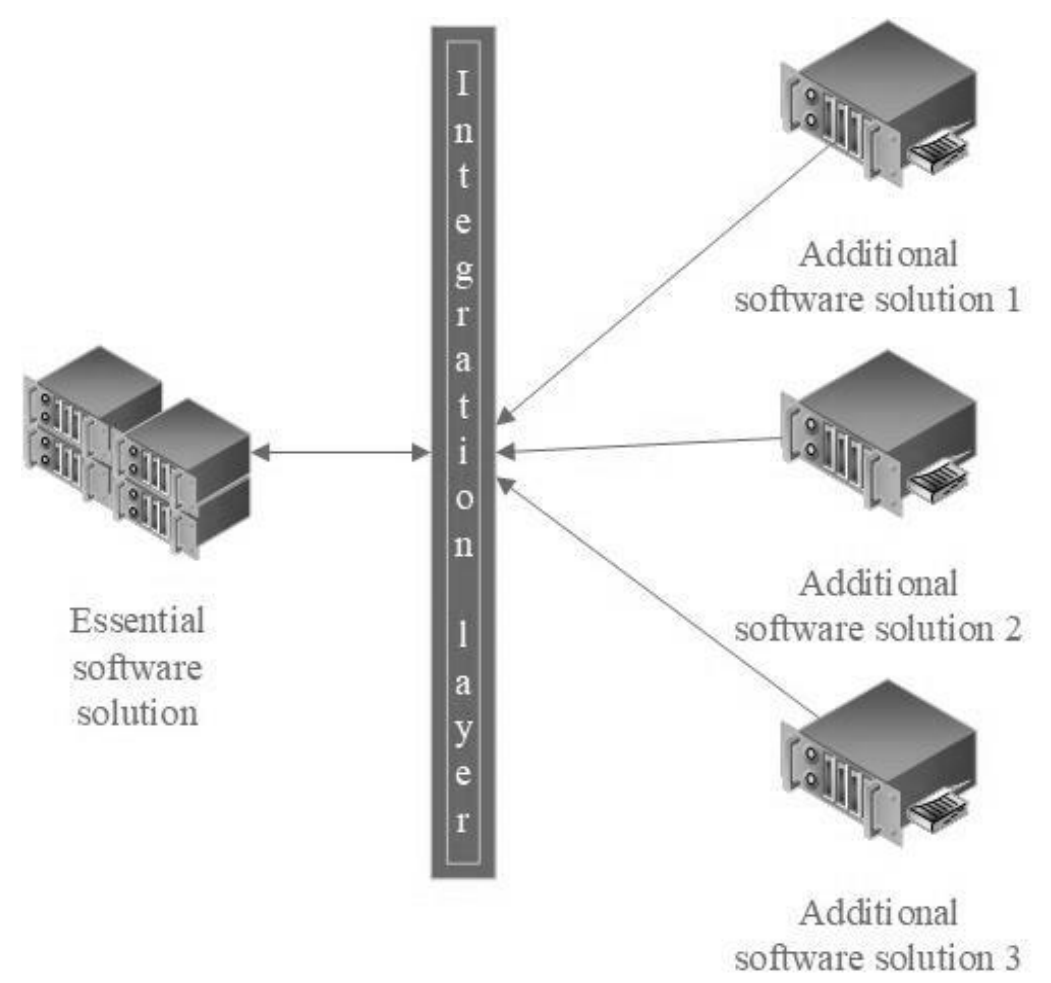

Figure 1. Integration of software solutions via an integration layer

Communication between individual software solutions takes place in any network environment using a communication protocol.
In today's global world and business necessity, this environment is usually the internet, and the protocol is TCP/IP. Figure 2 illustrates this approach. 


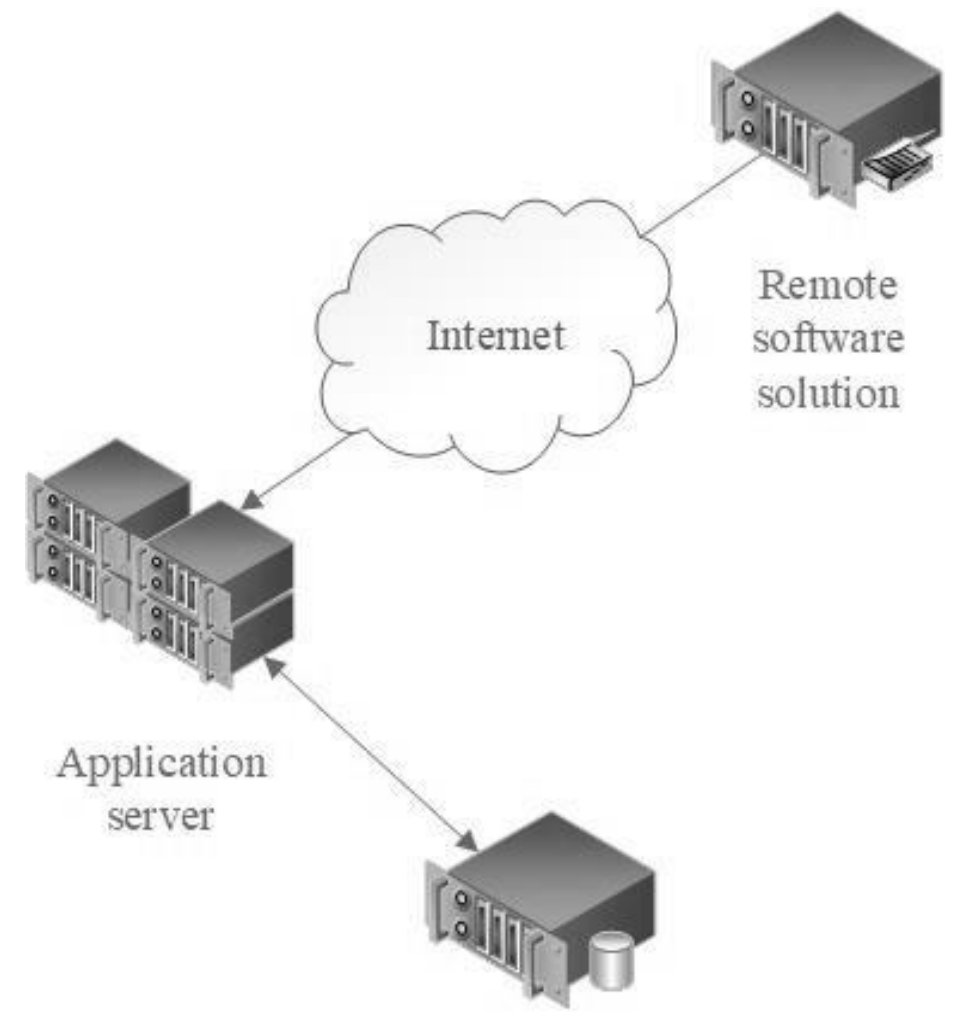

\section{Database}

server

Figure 2. Communication of software solutions through internet

For this integration to function properly, the remote software solution needs access to the attached information system server. In some cases, this will not be possible for technical and safety reasons. An example of such a case is the presence of a firewall that prevents access to the application server of the information system or filters that access by criteria that are unenforceable for the remote software solution.

\section{INTEGRATION VIA AN}

\section{INTERMEDIARY WEB SERVICE}

In cases where traditional approaches for integration of software solutions are not applicable, the integration needs to be realized in some other way. In the present study, this is an integration through the so-called intermediary service. The software as a service concept (SaaS) is a popular technology tool for exchanging business data between different software platforms for a long time. In recent years, however, the application of this concept has been explored in the context of the use of cloud services for expanding the capabilities of business information systems [9]. At the same time, the provision of services is also a major objective for the development of systems in the sphere of public administration and human resources $[10,11]$. Business, for its part, also relies heavily on similar solutions in the context of the need of the development of corporate data integration by external systems, including social networks [12]. Data integration is also very often associated with the need of aggregation of data for the purpose of business-specific information systems, including financial systems [13]. Data integration is being explored by many authors, with some emphasizing the need of using specific algorithms for systems with different business logic, including heterogeneous systems $[14,15]$. In any case, the application of the additional intermediary service in the integration process is an approach that deserves to be explored. Figure 3 illustrates this concept. With this approach, remote software solutions that want to communicate with the application server of the system are requesting the intermediary service. This service plays the role of a request distributor between the main software solution and the other (additional) software solutions. Intermediary service is logically separated into a different layer with its own access rules. In this way, it is always available from remote software solutions, regardless of the rules and constraints on the application server network 
connectivity. For the server, it is enough to only be able to receive requests from the intermediary web service, which facilitates security settings. For its part, the service is less pretentious about filtering incoming requests from additional software solutions. In this way,
MILEV P. system integration would also be feasible in an environment of specific network conditions, including in a web-based environment with a dynamic IP address or IP address that is not real.

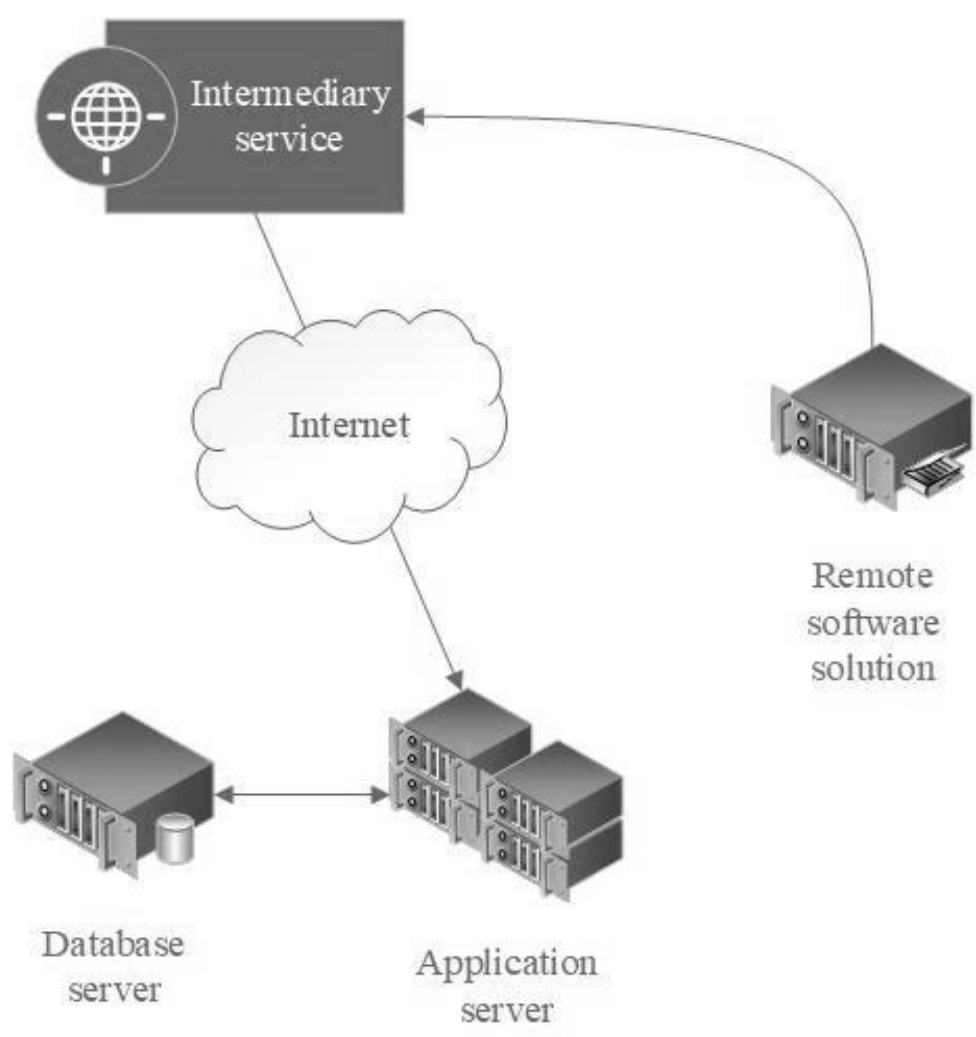

Figure 3. Integration of software solutions via an intermediary service

Thus, the integration of software solutions is more independent regarding the network specificities of the connection between the different components of the information system. Such integration is in many cases the only accessible way of communication to the application server of the system by the remote software solution.

\section{CONCLUSIONS}

In conclusion, the following results of the survey can be summarized:

- The traditional approaches for integration of software solutions have been clarified and this integration is usually realized with the help of an integration layer;

- The possibilities of integrating software solutions through the internet have been studied, which is the most common case of communication between software solutions for integration purposes;

- A model for the integration of software solutions has been defined with the help of an intermediary service.
The advantages of using the suggested in the article approach for integration of software solutions via an intermediary service can be presented in the following directions:

- The presence of an additional layer in the architecture of the software solution, which leads to a certain improvement in security in terms of access to the information system's data;

- Ability to further distribute business logic regarding the integration of the individual components of the software solution, which leads to an improvement in the processes of maintenance and development of the information system;

- Application of an internet-based architecture that has modern conceptual solutions.

\section{REFERENCES}

1. Belev, I., Software for Business Process Management and the Potential for Implementation in Bulgarian Universities, Economic and Social Alternatives, Issue 3, 2017, ISSN 1314-6556. 
2. Kirilova, K., Challenges to the Administrative Service of Municipalities in Bulgaria, Research Papers of UNWE, Issue 4, pp. 229-245, 2018, ISSN 0861-9344 (print), 2534-8957 (online).

3. Kirilov, R., Building E-Municipality, Problems, Approaches and Solutions, Trakia Journal of Sciences, Vol. 11, Suppl. 1, pp 58-63, 2013, ISSN 1313-7069 (print), 1313-3551 (online).

4. Stefanova, K., Kabakchieva, D., Analyzing University Data with Business Intelligence Systems for Management Decision Support, Economic and Social Alternatives, Issue 2, 2015, ISSN 1314-6556.

5. Marinova, N., Boychev, B., An analysis of the software solutions from the world's IaaS cloud services providers, Business Management, Issue 3, 2016, ISSN 25348396.

6. Murdjeva, A., Splitting Business Logic. Container for Analytical Business Logic, Research Papers of UNWE, Issue 2, pp. 235-276, 2008, ISSN 0861-9344 (print), 2534-8957 (online).

7. Kouzmanov, S., Analysis and Classification of Business Software Systems Integration Approaches, Economic Alternatives, Issue 2, 2015, ISSN 1312-7462.

8. Kirilov, R., Software Solutions for Managing Projects co-financed under the European Union's Operational Programmes, Business Management, Issue 3, 2016, ISSN 0861-6604.
9. Lazarova, V., The Expansion of the Ebusiness Information Systems in the Cloud - Problems and Solutions, Economic and Social Alternatives, Issue 4, 2015, ISSN 1314-6556.

10.Kirilova, K., Methodological Issues in Assessing the Capacity of Local Authorities for Developing and Delivering Electronic Services, Trakia Journal of Sciences, Vol. 11, Suppl. 1, pp. 85-88, 2013, ISSN 13137069 (print), 1313-3551 (online).

11.Daskalova, T., The human resources in the administration of "one-stop-shop", The Human Resources in the Panorama of Labor: a magazine for labor and social relations, Issue 2, pp. 26-35, 2009, ISSN 1312-305X.

12.Georgiev, V., Directions for Development of Corporate Communications through Social Network Tools, Economic and Social Alternatives, Issue 3, 2014, ISSN 1314-6556.

13.Amallah Binhaq, A., Design Platform Financial Aggregator in Agriculture, JSC, Vol. 2 (1), pp. 34-46, 2019, ISSN 26228254.

14.Tsaneva, M., A Practical Approach for Integrating Heterogeneous Systems, Business Management, Issue 2, 2019, ISSN 0861-6604.

15.Stefanov, G., Methods for Heterogeneity Detection During Multi-Dimensional Data Mart Integration, Economic Alternatives, Issue 1, 2015, ISSN 1312-7462. 